1 Title:

2 Frequency of a nucleotide overhang at the 5' end of hemorrhagic fever

3 mammarenavirus genomes in public sequence data

\title{
5 Authors:
}

6 Yutaro Neriya ${ }^{\mathbf{1}}$, Shohei Kojima ${ }^{2}$, Mai Kishimoto ${ }^{3}$, Arata Sakiyama ${ }^{4}$, Takao Iketani,

7 Tadashi Watanabe ${ }^{6}$, Yuichi Abe $^{7}$, Hiroshi Shimoda ${ }^{8}$, Keisuke Nakagawa ${ }^{9}$, Takaaki

$8 \mathrm{Koma}^{10}$, Yusuke Matsumoto ${ }^{11}$ \#

\section{Affiliations:}

$11{ }^{1}$ School of Agriculture, Utsunomiya University, 350 Mine-machi, Utsunomiya, Tochigi, 12 321-8505, Japan.

13 E-mail: neriya@cc.utsunomiya-u.ac.jp

2 Genome Immunobiology RIKEN Hakubi Research Team, RIKEN Center for

16 Integrative Medical Sciences, 1-7-22 Suehiro-cho, Tsurumi-ku, Yokohama, Kanagawa

17 230-0045, Japan.

18 E-mail: shohei.kojima@riken.jp

203 Division of Molecular Pathobiology, International Institute for Zoonosis Control, 21 Hokkaido University, North 20, West 10 Kita-ku, Sapporo 001-0020, Japan.

22 E-mail: kishimoto@czc.hokudai.ac.jp

$24{ }^{4}$ Department of Bacteriology, Osaka City University, Graduate School of Medicine, 25 1-4-3, Asahi-machi, Abeno-ku, Osaka 545-8585, Japan.

26 E-mail: sakiyama.arata@med.osaka-cu.ac.jp 
285 Laboratory of Pathogenesis of Viral Infectious Diseases, Tokyo University of

29 Agriculture and Technology, 3-8-1 Saiwai-chou, Fuchu, Tokyo 183-8509, Japan

$30 \quad$ E-mail: s215715r@st.go.tuat.ac.jp

${ }^{6}$ Department of Virology, Graduate school of medicine, University of the Ryukyus,

33 Uehara 207, Nishihara-cho, Nakagami-gun, Okinawa 903-0215, Japan.

34 E-mail: twatanab@med.u-ryukyu.ac.jp

7 Division of Molecular Diagnostics, Aichi Cancer Center Research Institute, 1-1,

37 Kanokoden, Chikusa-ku, Nagoya, 464-8681, Japan.

38 E-mail: y.abe@aichi-cc.jp

8 Laboratory of Veterinary Microbiology, Joint Faculty of Veterinary Medicine,

41 Yamaguchi University. 1677-1 Yoshida, Yamaguchi, 753-8515, Japan.

42 E-mail: hshimoda@yamaguchi-u.ac.jp

${ }^{9}$ Laboratory of Veterinary Microbiology, Joint Department of Veterinary Medicine,

45 Faculty of Applied Biological Sciences, Gifu University, 1-1 Yanagido, Gifu, 501-1193, 46 Japan.

47 E-mail: nkgwk@gifu-u.ac.jp

${ }^{10}$ Department of Microbiology, Tokushima University Graduate School of Biomedical

50 Sciences, 3-18-15 Kuramoto, Tokushima, 770-8503, Japan.

$51 \quad$ E-mail: tkoma@tokushima-u.ac.jp

55 E-mail: matsumoto-ys @igakuken.or.jp 
bioRxiv preprint doi: https://doi.org/10.1101/2022.02.04.479072; this version posted February 5, 2022. The copyright holder for this preprint (which was not certified by peer review) is the author/funder, who has granted bioRxiv a license to display the preprint in perpetuity. It is made available under aCC-BY 4.0 International license.

$56 \quad$ \# Author to whom correspondence should be addressed.

57 


\section{Abstract}

59 Mammarenaviruses, such as Lassa virus and South American hemorrhagic fever

60 (SAHF) virus, cause severe hemorrhagic fevers in humans, and pose major threats to

61 public health. Mammarenaviruses consist of a bi-segmented negative-sense RNA

62 genome in which the 5' and 3' ends form complementary strands that serve as a

63 replication promoter. Some mammarenaviruses have a nucleotide overhang at the 5',

64 genome end. By examining the complementarity of 5' and 3' genome ends using public

65 mammarenavirus genome sequences, we found that the 5' guanine overhang (5'-G

66 overhang) was present more frequently in Lassa and SAHF viruses than in other viruses.

67 The 5'-G overhang in the Lassa and SAHF virus sequences was found to be restricted to

68 the $\mathrm{L}$ and $\mathrm{S}$ segments, respectively. If the genome end sequence data in the public

69 database are accurate, the 5'-G overhang may be related to the high pathogenicity of

70 mammarenaviruses in humans.

71

72 Keywords: Mammarenaviruses, replication promoter, overhang, public sequence data 


\section{Introduction}

Arenaviruses (genus Mammarenavirus, family Arenaviridae, order Bunyavirales) are divided into Old World and New World complexes (1). The most significant pathogen in the Old World complex is Lassa virus, the cause of hemorrhagic Lassa fever in humans. Lymphocytic choriomeningitis virus (LCMV) is also an Old World arenavirus that can cause a serious neurological disease called lymphocytic choriomeningitis, especially in prenatal and immunocompromised humans (2). The New World arenaviruses are divided into three major clades (A, B, and C), with clade B containing the Junín, Machupo, Guanarito, and Sabiá viruses, which cause Argentinian, Bolivian, Venezuelan, and Brazililian hemorrhagic fevers, respectively; collectively, these are referred to as South America Hemorrhagic fever (SAHF) (3). The mammarenavirus genome consists of two negative-strand RNA segments encoding four viral proteins: a large (L) RNA-dependent RNA polymerase (RdRp) and a matrix protein $(\mathrm{Z})$ within the large RNA segment (L segment), and a nucleocapsid protein (NP) and a glycoprotein precursor (GPC) within the small RNA segment (S segment). The genomic RNA is encapsidated by NP and bound at both genome ends by L protein to form pseudocircularized ribonucleoprotein complexes (RNPs) (4-6). The genomic RNPs are enveloped by a lipid bilayer decorated with mature viral glycoproteins to form infectious virions.

In mammarenaviruses, the genome ends exhibit terminal complementarity of the 5' and 3' ends in each of the $\mathrm{L}$ and $\mathrm{S}$ segments to form a replication promoter. The interaction of each 5' and 3' end to L protein is essential for the initiation of RNA synthesis $(7,8)$. The 5 ' end of some mammarenavirus genomes contains an additional nontemplated guanine residue (5'-G overhang) (9-12), which has been considered to be generated by a prime-and-realign mechanism. During mammarenavirus replication, it is thought that RdRp initiates at position +2 of the promoter to generate a GC dinucleotide primer that realigns, leading to base pairing between the $\mathrm{C}$ and $\mathrm{G}$ at position +1 of the template (prime-and-realign) (13). Subsequent elongation by RdRp results in the 
102 synthesis of antigenomic RNPs with a pseudotemplated G residue at the 5' end (5'-G

103 overhang). The antigenomic RNPs are used as templates for mRNA synthesis, and the

$104 \mathrm{GPC}$ and $\mathrm{Z}$ mRNAs are transcribed from the $\mathrm{S}$ and $\mathrm{L}$ segments, respectively. The

105 antigenomic RNPs also serve as templates for GC-primed replication to produce

106 progeny genomic RNPs with 5'-G overhang. The mammarenavirus replication has been

107 also shown to be initiated with $\mathrm{CG}$ primer that starts with precise genomic end (+1

108 position) without generating $5^{\prime}-\mathrm{G}$ overhang (13). It remains unclear whether the

109 initiation of RNA synthesis and the genomic terminal structures are common between

110 all mammarenaviruses, and the biological significance of the 5'-G overhang has not

111 been studied in detail.

112 In this study, we constructed a list of mammarenavirus promotors by using public

113 sequence data to characterize the promoter structures of various species of

114 mammarenaviruses. We examined the conservation of sequences of promoters among

115 mammarenaviruses, and analyzed the genomic sequences for the presence of 5'- and

116 3'-overhangs.

\section{Methods}

\section{List of mammarenavirus promoters}

120 Forty-six mammarenavirus species were registered on the International 121 Committee on Taxonomy of Viruses (ICTV) list (https://talk.ictvonline.org/) on 122 December 7th, 2021. The complete sequences of S and L segments of 123 mammarenaviruses available on the National Center for Biotechnology Information 124 (NCBI) associated with Genbank accession numbers as listed in Supplementary Table 1 125 were used for the analysis. The sequences of the extreme 40 nucleotides (nts) of each of 126 the 5' and 3' ends of mammarenaviruses were collected, and the complementarity 127 between the 5' and 3' ends of the sequences was examined. The counts of G:C and A:U 128 complementarity and each of the nts (A, U, G, and C) in the promoter region are shown 
129 in Supplementary Table 1. The conservation of the nts in the promoter was analyzed by

130 using a sequence logo generator, Weblogo (https://weblogo.berkeley.edu/logo.cgi).

\section{Analysis of the 5'-overhang in the sequences of the Arenaviridae family}

132 Sequences annotated as Arenaviridae were downloaded from the NCBI refseq 133 database on January 7th, 2022. There were 5,274 Arenaviridae sequences in the 134 database. Sequences shorter than $1,000 \mathrm{nt}(\mathrm{n}=2,343)$ were omitted from this analysis.

135 We first checked for the presence of the promoter sequence, CGCA, in the 8-nt ends of 136 the sequences. The promoter sequence was present in both ends of 502 sequences. Then, 137 the overhangs were detected from the 502 sequences by a custom Python script. The 138 codes used for this analysis is available on GitHub 139 (https://github.com/shohei-kojima/Arenaviridae_overhang_analysis_2022).

\section{Results and Discussion}

\section{Construction of a list of promoters in mammarenaviruses}

143 To examine the conservation of the promoter sequence among virus species, we 144 compared the both extreme $40 \mathrm{nts}$ of the mammarenavirus genome sequences registered 145 on the ICTV list. Some mammarenavirus sequences available on the NCBI database did 146 not have complete genomic terminal information $(14,15)$. Sequences that showed 147 complementarity between +1 and +4 within 0 to 2 nt shifting of the 5' or 3 ' ends for 148 both L and S segments were selected (Figure 1A). We obtained 23 mammarenavirus 149 promoter sequences of both the L and S segments, as listed in Supplementary Table 1 150 (shown as the positive-strand antigenomic forms). The conservation of the extreme 38 151 nts (without the overhang) of each of the 5' and 3' ends of the L and S segments among 152 the 23 virus species was analyzed by using a sequence logo generator, Weblogo (Figure 153 1B). The first 5 nts of the 5' and 3' ends were completely conserved in both the L and S 154 segments in the 23 mammarenaviruses. Although the $+6 \mathrm{nt}$ in the 3 ' end varied in the L 155 segment, that in the S segment was $\mathrm{U}$ in almost all samples. This difference may result 156 in differences in the promoter strength of the L and S segments to create these segments 
157 in a different ratio in infected cells as proposed in other segmented RNA viruses $(16,17)$.

158 To further characterize the promoter structure, the counts of G:C and A:U

159 complementarity and A, U, G, and C in the first 40 nts (for 38 to 39 nts in the opposite

160 strand of 2-nt and 1-nt overhangs, respectively) of the L and S segments were

161 determined (Figure 1C and Supplementary Table 1). G:C complementarity was

162 significantly higher than A:U complementarity in both the L and S segments; this result

163 differed from that of tri-segmented viruses belonging to the order Bunyavirales, which

164 showed higher A:U complementarity in the promoters (18). The L and S segments

165 contained significantly less $\mathrm{A}$ than $\mathrm{U}, \mathrm{G}$, and $\mathrm{C}$ in the $5^{\prime}$ ends, which results in an $\mathrm{nt}$

166 bias between the genomic and antigenomic promoters. This may function to regulate the

167 replication balance between genome and antigenome to produce different ratios of

168 various viral RNA species as observed in infected cells (19). The 5'-CGCA-UGCG-3'

169 sequence was the most conserved genome end sequence in the 23 mammarenaviruses

170 (Figure 1D, non-overhang). The 5'-GCGCA-UGCG-3' sequence showed the presence

171 of an unpaired 5' guanine nt (Figure 1D, 5'-G overhang). Among the 23 viruses, the

172 5'-G overhang was found in the Lassa, Guanarito, Junín, Machupo, Sabiá, and Oliveros

173 viruses (Figure 1D), and the 2-nt 3'-overhang was found only in the Lunk virus S

174 segment (Supplementary Table 1) in the public database. All viruses, except for

175 Oliveros virus $(20,21)$, with the 5'-G overhang are associated with human hemorrhagic

176 fever, and are categorized as biosafety level 4 pathogens.

177 Analysis for the 5'-G overhang in the Arenaviridae family using public sequence

178 data

179 To expand the study of viruses having the 5'-G overhang, we next analyzed 180 sequences with 5'-CGCA-UGCG-3' (non-overhang), 5'-GCGCA-UGCG-3' (5'-G

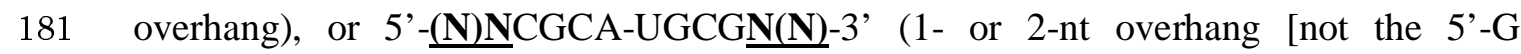
182 overhang]) in sequences of the Arenaviridae family (Figure 2A). Among the virus 183 sequences, artificially generated sequences (e.g., generated by reverse genetics virus 184 engineering) were excluded. We found that 345 sequences did not have the overhang 
185 (5'-CGCA-UGCG-3'), whereas 58 sequences had the 5'-G overhang

186 (5'-GCGCA-UGCG-3'; Supplementary Table 2). The sample numbers of over 5

187 sequences per virus species are shown in Figure 2B. We found that virus genomes with

188 the 5'-G overhang were restricted to the Lassa, LCMV, Junín, Machupo, Guanarito,

189 Sabiá, and Oliveros viruses (Figure 2B and C). All LCMVs with the 5'-G overhang

190 found in this study were clones obtained from virus-inoculated laboratory mice $(22,23)$.

191 We next determine which segments ( $\mathrm{L}$ or S) contained the 5'-G overhang in the Lassa,

192 Junín, Machupo, Guanarito, Sabiá, and Oliveros viruses. Guanarito virus sequences had

193 the 5'-G overhang only in the L segment, Lassa and Oliveros virus sequences had the

194 5'-G overhang only in the S segment, and the other sequences had it in both segments

195 (Figure 2D and E). L segments with the 5'-G overhang were found only in the New

196 World viruses causing SAHF. In general, the Old World viruses are geographically

197 confined to the African continent, with the exception of LCMV that circulates globally.

198 The New World viruses are found on the South and North American continents.

199 Regardless of the region where the viruses are distributed, the 5'-G overhang was found

200 specifically in hemorrhagic fever virus sequences on both continents. Although Oliveros

201 virus is a New World virus, it had the 5'-G overhang in the L segment, unlike other

202 SAHF viruses. This slight difference in the Oliveros virus from other SAHF viruses

203 might be the reason for its non-pathogenic characteristic.

\section{Minor genome end sequences in the database}

205 Among the 447 sequences described in Figure 2A, we found 35 sequences that did

206 not have 5'-CGCA-UGCG-3' (non-overhang) or 5'-GCGCA-UGCG-3' (5'-G

207 overhang). Two minor non-overhang sequences, i.e., 5'-GCGCA-UGCGㅡ-3' and

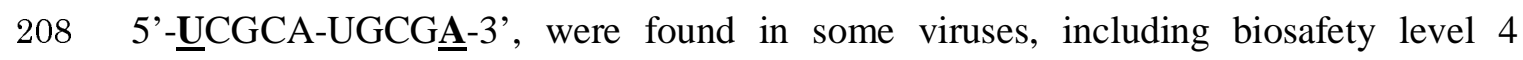

209 pathogens (5' - GCGCA-UGCG $\underline{\mathbf{C}}-3^{\prime}$ in Lassa, Lujo, and Junín viruses, and

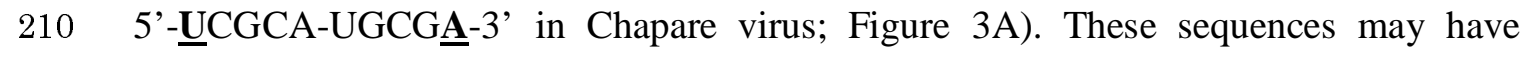

211 evolved from the 5' overhang with an additional nt on the opposite end of the overhang.

212 We also found some variations in the 5' overhang (not the 5'-G overhang; Figure 3B). 


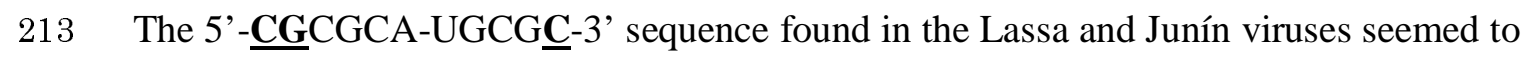

214 be a 1-nt addition to the 5' end of the minor non-overhang shown in Figure 3A. The 215 3'-C overhang (5'-CGCA-UGCGㅡ-3') was found in the Lassa, Oliveros, and other 216 virus sequences (Figure 3B). When viral replication occurs using the genome with a 217 5'-G overhang as a template, the 3' end of the newly replicated RNA terminates with a 218 C complementary to the overhanging G. It has been presumed that mammarenaviruses 219 remove this last $\mathrm{C}$ to retain genome integrity (13). The 3'-C overhang may represent 220 this $\mathrm{C}$ that was not removed during the replication process. The 3 ' overhang 221 (5' - GCGCA-UGCG $\underline{\mathbf{C G}-3}$ ') found in Lassa virus (Figure 3C) was also considered to 222 have arisen from a minor non-overhang. There were a few virus sequences possessing a 223 2-nt overhang both in the 5' and 3' ends, i.e., an unidentified arenavirus sp. and Lunk 224 virus (Figure 3B and $\mathrm{C}$ ). The mechanism of their generation and their biological 225 properties remain unknown.

\section{Dependence on the accuracy of the sequence database}

227 All of the results of this study depend on the accuracy of the public genome 228 sequences. If the genome end sequences in the NCBI database were correct and 229 accurately registered, hemorrhagic fever viruses appear to have a higher frequency than 230 other non-pathogenic arenaviruses of containing the 5'-G overhang in their genomes. 231 However, some of the sequences in the database were obtained from full-genome 232 amplification via reverse transcription polymerase chain reaction, and in those cases, the 233 extreme sequences are derived from oligonucleotide primers. Further experiments are 234 needed to validate whether the $5^{\prime}-\mathrm{G}$ overhang correlates with pathology. Even if a 235 mutant arenavirus that has an artificially attached or removed 5'-G overhang is 236 produced using reverse genetics, it would be difficult to evaluate whether changes in its 237 pathogenicity are due to the presence or absence of the 5'-G overhang, because the virus 238 can automatically synthesize the overhang by the prime-and-realign mechanism of the L 239 protein. Identification of the functional region in the L protein that is involved in the 
240 prime-and-realign mechanism and that confers a 5' $-\mathrm{G}$ overhang is essential to prove a

241 correlation between the 5'-G overhang and viral pathogenicity.

242 The advantage of the 5'-G overhang for arenavirus growth and pathogenesis

243 The high frequency of the 5'-G overhang in hemorrhagic fever virus sequences in 244 the public database suggests that hemorrhagic fever viruses have a higher ability than 245 other viruses to produce the overhang via prime-and-realign. It was hypothesized that 246 the 5'-G overhang might increase the pathogenicity of these viruses in humans. It has 247 been proposed that the 5'-G overhang in the promoter protects this portion of 248 double-stranded RNA from being recognized by retinoic acid-inducible gene I (24). The

249 presence of the 5'-G overhang was shown to be important for stimulating the RNA 250 synthesis of purified Machupo and Lassa virus L protein $(25,26)$. These facts indicate 251 that the presence of the 5'-G overhang may be an important factor associated with viral 252 pathogenicity via the enhancement of host immune evasion and polymerase activity.

\section{$254 \quad$ Funding}

255 This work was supported by Takeda Science Foundation, and Tokyo Biochemical 256 Foundation, Japan (to Y.M.).

Author contributions and designed the study, performed the analyses, analyzed the data. Y.M. wrote the manuscript. All authors have read and agreed to the manuscript.

264 The authors declare no competing interests. 


\section{Reference}

1. Hallam SJ, Koma T, Maruyama J, Paessler S. Review of mammarenavirus biology and replication. Front Microbiol. 2018 9:1751.

2. Bonthius DJ. Lymphocytic choriomeningitis virus: An under-recognized cause of neurologic disease in the fetus, child, and adult. Semin Pediatr Neurol. 2012 19(3):89-95.

3. Spiropoulou CF, Kunz S, Rollin PE, Campbell KP, Oldstone MBA. New World Arenavirus clade $\mathrm{C}$, but not clade $\mathrm{A}$ and $\mathrm{B}$ viruses, utilizes $\alpha$-dystroglycan as its major receptor. J Virol. 2002 76(10):5140-6.

4. Vezza AC, Clewley JP, Gard GP, Abraham NZ, Compans RW, Bishop DH. Virion RNA species of the arenaviruses Pichinde, Tacaribe, and Tamiami. J Virol. 1978 26(2):485-97.

5. Young PR, Howard CR. Fine structure analysis of Pichinde virus nucleocapsids. J Gen Virol. 1983 64:833-42.

6. Young PR, Howard CR. Ribonucleoprotein complexes associated with virions of Pichinde virus and Pichinde virus-infected cells. Med Microbiol Immunol (Berl). 1986 175(2-3):79-83.

7. Kouba T, Vogel D, Thorkelsson SR, Quemin ERJ, Williams HM, Milewski M, Busch C, Günther S, Grünewald K, Rosenthal M, Cusack S. Conformational changes in Lassa virus $\mathrm{L}$ protein associated with promoter binding and RNA synthesis activity. Nat Commun. 2021 12(1):7018.

8. Peng R, Xu X, Jing J, Wang M, Peng Q, Liu S, Wu Y, Bao X, Wang P, Qi J, Gao GF, Shi Y. Structural insight into arenavirus replication machinery. Nature. 2020 579(7800):615-9. 
291 9. Garcin D, Kolakofsky D. A novel mechanism for the initiation of Tacaribe 292 arenavirus genome replication. J Virol. 1990 64(12):6196-203.

293
10. Polyak SJ, Zheng S, Harnish DG. 5' termini of Pichinde arenavirus S RNAs and mRNAs contain nontemplated nucleotides. J Virol. 1995 69(5):3211-5.

11. Auperin DD, Sasso DR, McCormick JB. Nucleotide sequence of the glycoprotein gene and intergenic region of the Lassa virus S genome RNA. Virology. 1986 154(1):155-67.

12. Gonzalez JPJ, Bowen MD, Nichol ST, Rico-hesse R. Genetic characterization and phylogeny of Sabiá virus, an emergent pathogen in Brazil. Virology. 1996 221(2):318-24.

13. Garcin D, Kolakofsky D. Tacaribe arenavirus RNA synthesis in vitro is primer dependent and suggests an unusual model for the initiation of genome replication. J Virol. 1992 66(3):1370-6.

14. Holzerland J, Leske A, Fénéant L, Garcin D, Kolakofsky D, Groseth A. Complete genome sequence of Tacaribe virus. Arch Virol. 2020 165(8):1899-903.

15. Ye C, de la Torre JC, Martínez-Sobrido L. Development of reverse genetics for the prototype New World mammarenavirus Tacaribe virus. J Virol. 2020 94(19):e01014-20.

16. Matsumoto Y, Ohta K, Kolakofsky D, Nishio M. A minigenome study of Hazara nairovirus genomic promoters. J Virol. 2019 93(6):e02118-18.

17. Barr JN, Wertz GW. Bunyamwera Bunyavirus RNA synthesis requires cooperation of 3'- and 5'-terminal sequences. J Virol. 2004 78(3):1129-38. 
313 18. Ferron F, Weber F, de la Torre JC, Reguera J. Transcription and replication mechanisms of Bunyaviridae and Arenaviridae L proteins. Virus Res. 2017 234:118-34.

19. King BR, Samacoits A, Eisenhauer PL, Ziegler CM, Bruce EA, Zenklusen D,

20. Bowen MD, Peters CJ, Mills JN, Nichol ST. Oliveros Virus: A Novel Arenavirus from Argentina. Virology. 1996 217(1):362-6.

21. Mills JN, Barrera Oro JG, Bressler DS, Childs JE, Tesh RB, Smith JF, Enria DA, Geisbert TW, McKee KT Jr, Bowen MD, Peters CJ, Jahrling PB. Characterization of Oliveros virus, a new member of the Tacaribe complex (Arenaviridae: Arenavirus). Am J Trop Med Hyg. 1996 54(4):399-404.

22. Chen M, Lan S, Ou R, Price GE, Jiang H, de la Torre JC, Moskophidis D. Genomic and biological characterization of aggressive and docile strains of lymphocytic choriomeningitis virus rescued from a plasmid-based reverse-genetics system. J Gen Virol. 89(6):1421-33.

23. Pfau CJ, Valenti JK, Pevear DC, Hunt KD. Lymphocytic choriomeningitis virus killer T cells are lethal only in weakly disseminated murine infections. J Exp Med. 1982 156(1):79-89.

24. Marq J-B, Kolakofsky D, Garcin D. Unpaired 5' ppp-nucleotides, as found in arenavirus double-stranded RNA panhandles, are not recognized by RIG-I. J Biol Chem. 2010 285(24):18208-16. 
337 25. Pyle JD, Whelan SPJ. RNA ligands activate the Machupo virus polymerase and 338 guide promoter usage. Proc Natl Acad Sci U S A. 2019 116(21):10518-24.

339 26. Vogel D, Rosenthal M, Gogrefe N, Reindl S, Günther S. Biochemical 340 characterization of the Lassa virus L protein. J Biol Chem. 2019 $341 \quad 294(20): 8088-100$. 
343 Figure 1. Comprehensive analysis of the genomic promoters in mammarenaviruses

344 (A) Schematic diagram of mammarenavirus promoter sequences and the 345 complementarity of the 5' and 3' ends. (B) The sequence conservation of the $38 \mathrm{nts}$ in 346 the 5' and 3' genome ends of the $\mathrm{L}$ and $\mathrm{S}$ segments (non-overhang region) among 23 347 mammarenavirus species was analyzed by a sequence logo generator, Weblogo. (C) 348 Counts of G:C and A:U complementarity and each nt in the first 40 nts. Bars represent 349 the means and statistical deviations. Inferential statistical analysis was performed by 350 one-way analysis of variance (ANOVA) followed by the Tukey's test. *p $<0.01$, **A $>$ $351 \mathrm{U}, \mathrm{G}$, and $\mathrm{C}$ in 5 ' end $(\mathrm{p}<0.05)$, *** $\mathrm{A}>\mathrm{U}, \mathrm{G}$, and $\mathrm{C}$ in 3 ' end $(\mathrm{p}<0.05)$. (D) 352 Schematics of the first 4 to 5 nts in the non-overhang and 5'-G overhang genome ends. 353 Virus species possessing the 5'-G overhang are shown. All sequences are shown as the 354 positive-strand antigenomic form.

Figure 2. Analysis of the overhang at the genome end in the Arenaviridae family

357 (A) Schematic diagram of the analysis of genome end sequences in the Arenaviridae 358 family. Viral antigenome sequences starting with 5'-CGCA and ending with UGCG-3' 359 were recognized as not having the overhang (non-overhang), and those starting with 360 5'-GCGCA and ending with UGCG-3' were recognized as having the 5'-G overhang. 361 (B) The number of sequences per virus species recognized as not having 362 (non-overhang) or having the 5'-G overhang shown in panel A. (C) The percentage of 363 sequences possessing the 5'-G overhang among the total sequences. HFV: hemorrhagic 364 fever virus. The full virus names are shown in Supplementary Table 2. Reptarena: 365 unidentified reptraena virus strains. (D) The number of sequences possessing the 5'-G 366 overhang in the L and S segments. (E) The percentage of sequences possessing the 5'-G 367 overhang in the $\mathrm{L}$ and $\mathrm{S}$ segments $(\mathrm{L}+\mathrm{S}=100 \%)$.

\section{Figure 3. Minor genome end sequences in the Arenaviridae family}



available under aCC-BY 4.0 International license.

370 (A) Minor sequences without the overhang (non-overhang). (B) Minor sequences with 371 the 5' overhang. (C) Sequences with the 3' overhang. Additional nts outside of the 372 5'-CGCA-UGCG-3' sequence are shown in green. HFVs are shown in red. 
Sequences of 46 mammarenaviruses registered on the ICTV list

Non-overhang

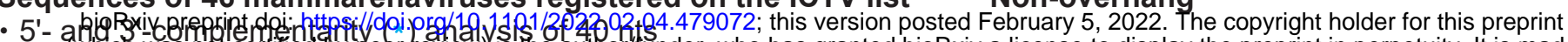

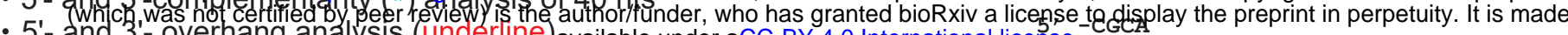

- 5'- and 3'- overhang analysis (underline) available under aCC-BY 4.0 International licen $3^{\prime}$.

5' -GCGCACCGGGGAUCCUAGGCAUUUUUGGUUGCGCAAUUCA

3' - GCGUGUCACCUAGGAUCCGAUAACCUAACGCGAAACGAA $+01234$

1

5'-G overhang

5' -GCGCA

$3^{\prime}-$ GCGU
Lassa virus

Guanarito virus Junín virus

Machupo virus

Sabiá virus

Oliveros virus

Data selection for analysis

1. Complement +1 to +4 nts

2. Complement +1 to +4 nts within 2 nt overhang

$\downarrow$

Complete list of promoters (Supplemmentary Table 1)

B
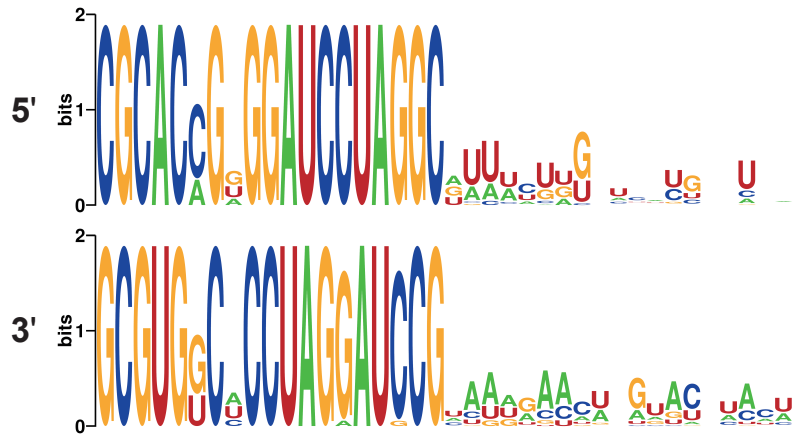

L segment
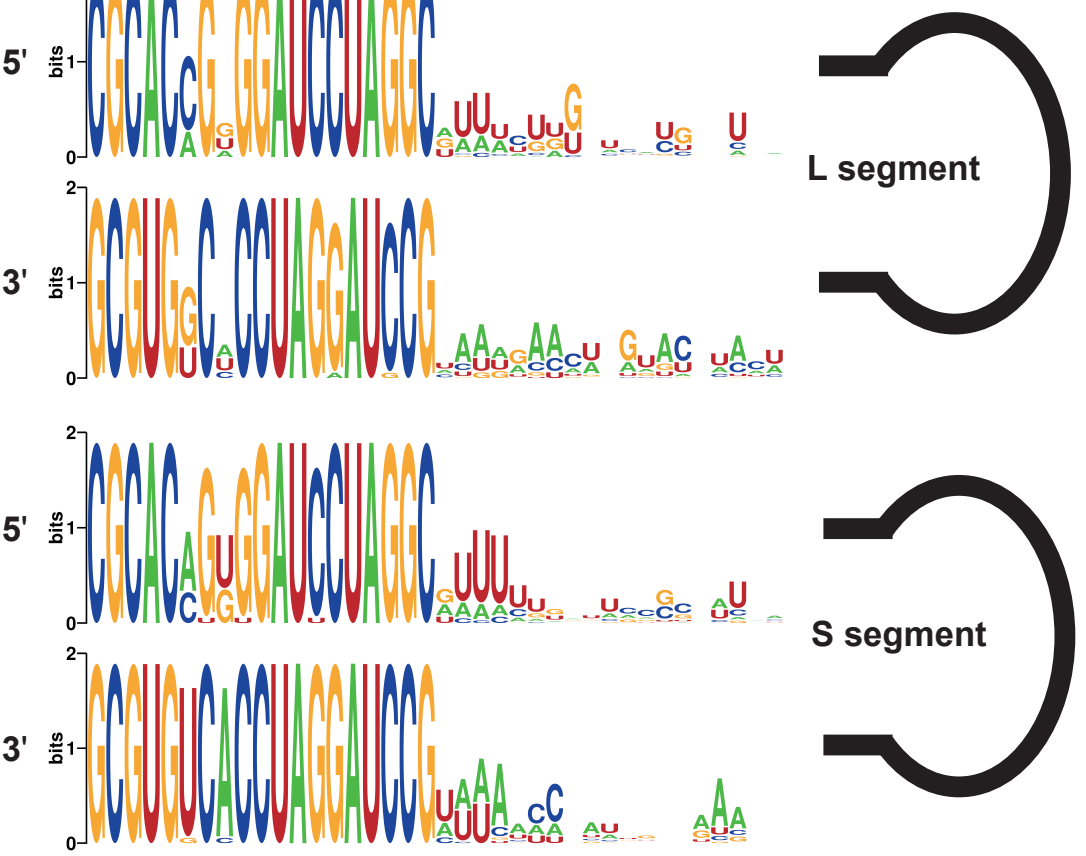

C
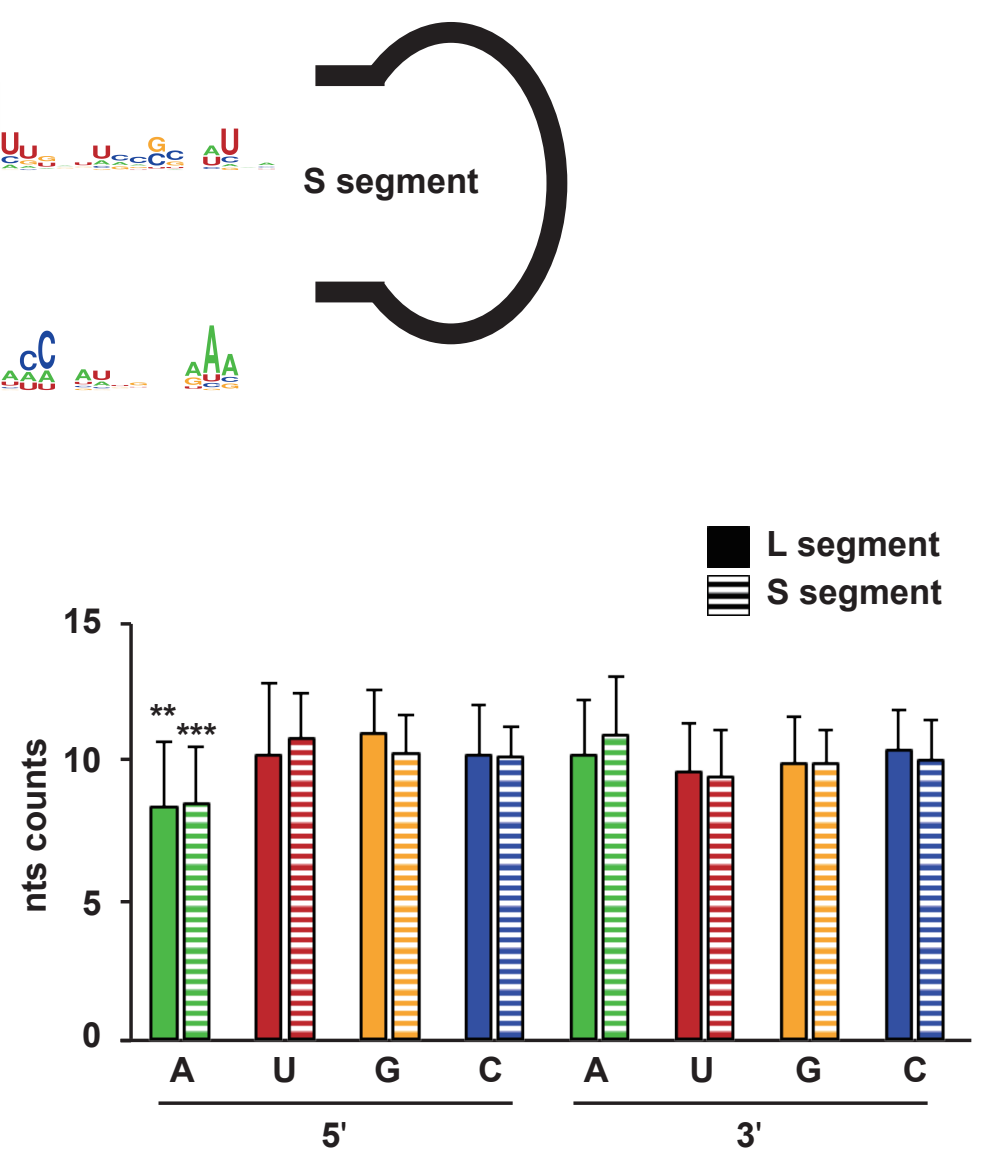

Figure 1

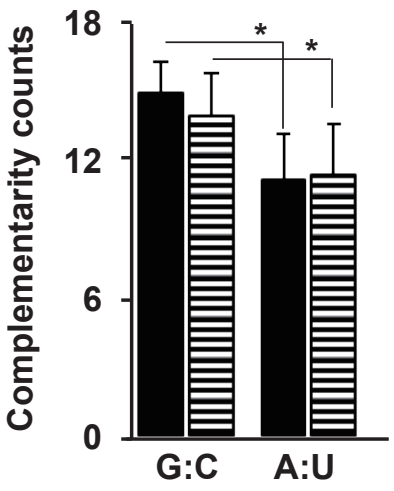


A

"Arenaviridae" 447 sequences

Analysis of genome ends

2022. The copyitgiseglment this preprint

$\begin{array}{lll}\text { Non-overhang } & 5^{\prime} & \text {-CGCA } \\ 3^{\prime} & - \text { GCGU }\end{array}$

5'-G overhang $\quad 5^{\prime}$-GCGCA

$3^{\prime}-$ GCGU

B

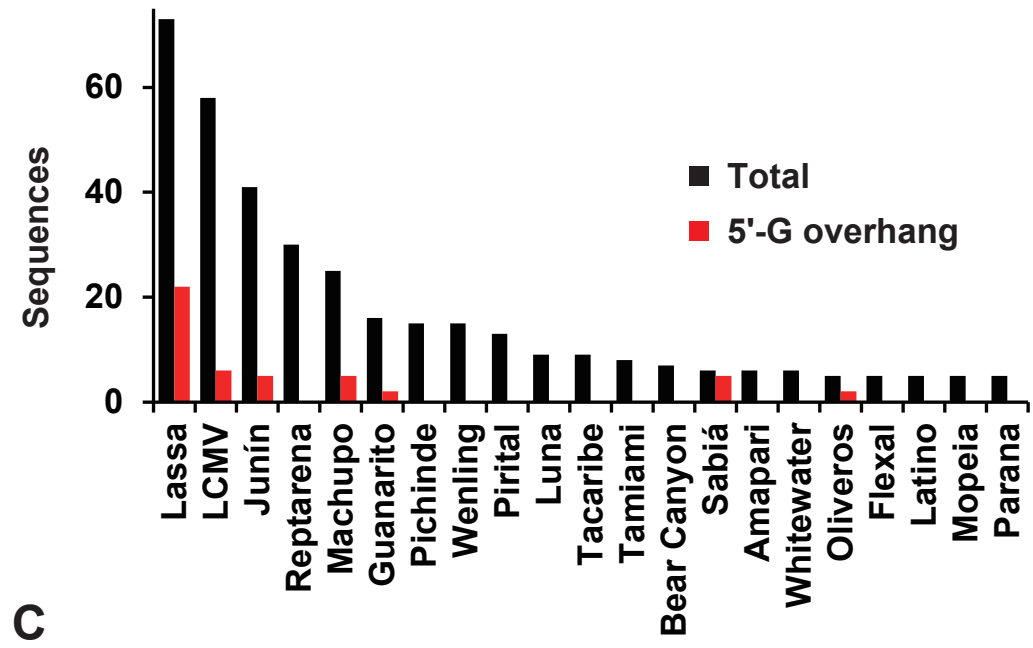

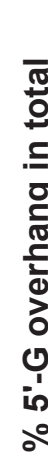

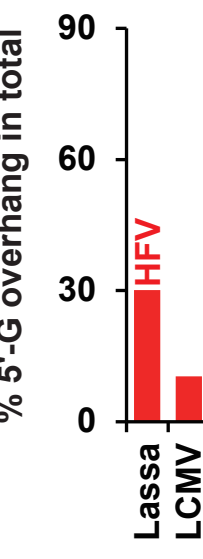

崖 available under aCC-BY 4.0 International licen

E
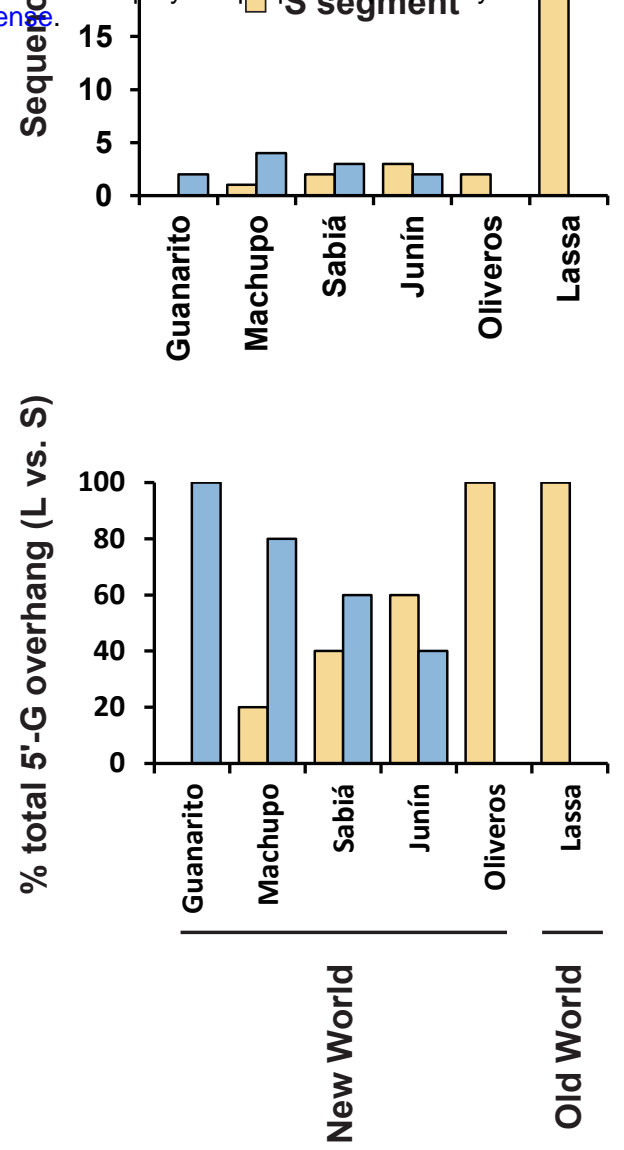

Figure 2 
bioRxiv preprint doi: https://doi.org/10.1101/2022.02.04.479072; this version posted February 5, 2022. The copyright holder for this preprint (which was not certified by peer review) is the author/funder, who has granted bioRxiv a license to display the preprint in perpetuity. It is made A Non-overhang

$\begin{array}{ll}\text { 5'-GCGCA } & \text { Lassa (4) } \\ \text { 3'-CGCGU } & \text { Lujo (3) } \\ & \text { Junín (1) } \\ & \text { Keijut (1) } \\ & \text { Luna (1) }\end{array}$

S5-like (1)

Univ of Giessen (1)

5'-UCGCA Chapare (2)

3'-AGCGU

B

\section{5' overhang}

5'-UCGCA

3'- GCGU

5'-CGCGCA

3'- CGCGU

5'-AUCGCA

3'- CGCGU

5'-UUCGCA

3'- AGCGU

5'-UGCGCA

3'- GCGU
LCMV (2)

Lassa (4)

Junín (1)

Guanarito (1)

Wehzhou (1)

Arena sp. (1)
C 3' overhang

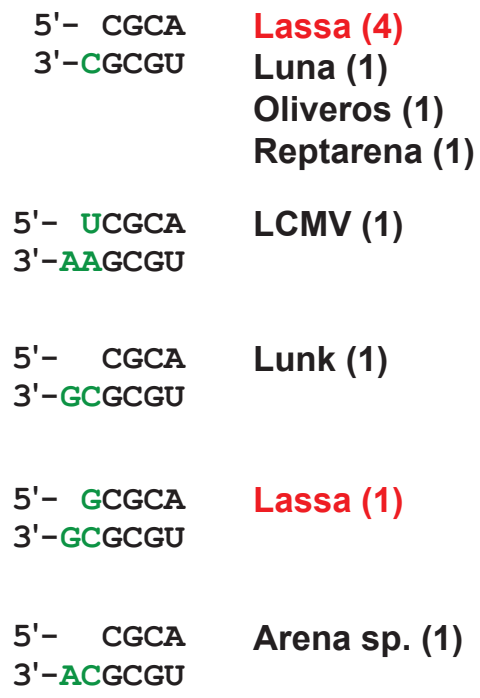

\section{Figure 3}

